

\title{
Generalized expressions of reliability of series-parallel and parallel-series systems using the Transferable Belief Model
}

Felipe Aguirre, Mohamed Sallak, Walter Schön

\section{- To cite this version:}

Felipe Aguirre, Mohamed Sallak, Walter Schön. Generalized expressions of reliability of series-parallel and parallel-series systems using the Transferable Belief Model. European Safety and Reliability Conference (ESREL 2011), Sep 2011, Troyes, France. pp.2137-2142. hal-00646300

\section{HAL Id: hal-00646300 \\ https://hal.science/hal-00646300}

Submitted on 29 Nov 2011

HAL is a multi-disciplinary open access archive for the deposit and dissemination of scientific research documents, whether they are published or not. The documents may come from teaching and research institutions in France or abroad, or from public or private research centers.
L'archive ouverte pluridisciplinaire HAL, est destinée au dépôt et à la diffusion de documents scientifiques de niveau recherche, publiés ou non, émanant des établissements d'enseignement et de recherche français ou étrangers, des laboratoires publics ou privés. 


\title{
Generalized expressions of reliability of series-parallel and parallel-series systems using the Transferable Belief Model
}

\author{
Felipe Aguirre \& Mohamed Sallak \& Walter Schön \\ Laboratoire Heudiasyc, UMR CNRS 6599. \\ Université de Technologie de Compiègne, France
}

\begin{abstract}
Probability theory is well suited to treat uncertainties when their origin comes only from the natural variability of components' failure (aleatory uncertainty). On the other hand, if the uncertainties are due to incompleteness, imprecision or ignorance of the reliability data (epistemic uncertainty), several theories can be used. Previous studies have shown that the Transferable Belief Model (TBM) which is an interpretation of the Dempster Shafer theory is a well suited framework to treat both types of uncertainties. Nevertheless, past experiences have proven that the computational cost of the TBM based model grows exponentially with the size of the system. To overcome this situation, generalized expressions were defined so as to have a more efficient way to study the systems' reliability of series-parallel and parallel-series configurations. Through the use of these expressions, there is no need to go by the TBM operations (conjunctive rule, extensions, etc.) to evaluate the overall system's reliability.
\end{abstract}

\section{INTRODUCTION}

Over the last two decades, many researchers have used other theories different from probability theory, in reliability and risk assessments to treat uncertainties.

There are different ways of classifying uncertainty. One of the most widely used is to divide it in two different types: aleatory uncertainty and epistemic uncertainty. The former, also called irreducible uncertainty, arises from natural stochasticity or environmental variation across space or through time. The latter, also called reducible uncertainty, arises from incompleteness or ignorance of knowledge or data (Oberkampf et al. 2004).

For many years, the probability theory has been used as the primary tool in reliability and risk assessments. However, in the presence of epistemic uncertainty, other theories are better suited than probability theory. Moreover, it has been proven that uncertainties in reliability and risk assessments are mainly epistemic (Drouin et al. 2009). The most promising theories to treat aleatory and epistemic uncertainty are:

- Probability bound analysis (Moore 1979).

- Upper and lower previsions (Walley 1991).

- Fuzzy set theory (Zadeh 1965).

- Possibility theory (Zadeh 1965).
- Dempster-Shafer theory (Dempster 1967, Shafer 1976).

From the above theories, the Dempster-Shafer (DS) theory has been proven as a well suited theory for the treatment of aleatory and epistemic uncertainty in the reliability analysis (Sallak et al. 2010, Aguirre et al. 2010), furthermore, it allows also the modelisation of failure dependencies between components. D-S theory generalizes both the probability theory and sets theory, even though the mechanics of operations in these three theories are completely different. The D-S theory has several interpretations: Dempsters model (Dempster 1967), the theory of hint (Kohlas and Monney 1995), the probability of modal propositions model (Ruspini 1986) and the Transferable Belief Model (TBM) (Smets and Kennes 1994). In this work, the TBM has been chosen because it is a model developed outside the scope of probability theory and avoids the criticisms that the D-S theory is understood as a special form of upper and lower probability theory (Aven 2010).

Past experiences have proven that the computational cost of the TBM based model grows rapidly with the size of the system. This is due to the fact that the cardinality of the domain where the resulting mass function is defined is equal to $3^{n}$, with $n$ being the size of the system. To overcome this situation, generalized expressions are introduced on the paper so as to have a more efficient way to study the reli- 
ability of systems. These expressions can be used to study series-parallel, parallel-series and combinations of series-parallel and parallel-series systems.

The expressions were obtained using the method of minimal cuts and the general expressions of reliability of series and parallel systems presented in (Sallak et al. 2010). The advantage of this equations is that any system that can be represented as a parallelseries or series-parallel system can be studied easily and faster. This way, there is no need to go by the TBM operations, like the conjunctive rule of combination, thus, avoiding the exponential growth of the TBM.

Section 2 presents an introduction to the TBM theory and explains the mechanics of the TBM reliability assessment model. Section 3 talks about the computational cost of the TBM based model and discusses possible optimization methods. Section 4 introduces the generalized expressions for series-parallel and parallel-series systems. Finally, section 5 exposes two applications of the expressions and section 6 finishes with some conclusions.

\section{TBM BASED RELIABILITY MODEL}

The TBM was introduced by Smets \& Kennes (1994) as a subjectivist interpretation of D-S theory. The D$\mathrm{S}$ theory, also called evidence theory, was first described by Dempster (1967) with the study of upper and lower probabilities and extended by Shafer (1976). The TBM represents a unique framework for representing and manipulating aleatory and epistemic uncertainties. In a finite discrete space, the TBM can be interpreted as a generalization of probability theory where probabilities can be assigned to any subsets instead of singletons only. In this section, basic notions, extended operations, and terminology of TBM are explained. For a more detailed exposition see (Smets \& Kennes 1994).

\subsection{Basic Probability Assignment (BPA)}

A Basic Probability Assignment (BPA) on the frame of discernment $\Omega$, also called Basic Belief Assignment (BBA), is a function, $m^{\Omega}: 2^{\Omega} \rightarrow[0,1]$, which maps belief masses not only on events but also on subsets of events (The power set $2^{\Omega}$ ), such that:

$\sum_{A \in 2^{\Omega}} m^{\Omega}(A)=1$

As an example, let's consider $\Omega=\left\{x_{1}, x_{2}, x_{3}\right\}$ as our frame of discernment. Then, $x_{1}, x_{2}$ and $x_{3}$ are elementary propositions and mutually exclusive to each other. Through the use of the TBM, a piece of evidence represented by a BPA allocates beliefs to the subsets belonging to the power set $2^{\Omega}=$ $\left\{\{\emptyset\},\left\{x_{1}\right\},\left\{x_{2}\right\},\left\{x_{3}\right\},\left\{x_{1}, x_{2}\right\},\left\{x_{1}, x_{3}\right\},\left\{x_{2}, x_{3}\right\}, \Omega\right\}$ in such a way that equation 2.1 is respected.
Every subset $A$ to which a belief different from zero is allocated $\left(m^{\Omega}(A) \neq 0\right)$ is called a focal element and this value represents the belief that the answer is in $A$ (Smets \& Kennes 1994). A BPA having a singleton $\{x\}(x \in \Omega)$ as a unique focal set represents full knowledge. A BPA having only singletons as focal sets is equivalent to probabilities. A BPA having $\Omega$ as a unique focal set represents complete ignorance and is called vacuous. In addition, BPAs has further properties, which distinguishes it from being a probability function:

- It is not required that $m(\Omega)=1$.

- It is not required that $m(A) \leq m(B)$ when $A \subseteq$ $B$.

- There is no relationship between $m(A)$ and $m(\bar{A})$.

- $m(A)+m(\bar{A})$ does not always have to be 1 .

\subsection{Conjunctive combination rule}

The conjunctive combination rule is used to combine two distinct pieces of evidence $m_{i}^{\Omega}$ and $m_{j}^{\Omega}$ that are reliable and come from two different sources $i$ and $j$ (Smets \& Kennes 1994). The rule is implemented using the following equation:

$$
m_{i \cap j}^{\Omega}(H)=\sum_{A \cap B=H} m_{i}^{\Omega}(A) m_{j}^{\Omega}(B), \forall A, B, H \subseteq \Omega
$$

Other combination rules exit (see Sentz \& Ferson 2002) but, in this paper, only the conjunctive rule of combination is needed

\subsection{Operations on Joint Spaces}

The marginalisation of a BPA $m^{\Omega_{x} \Omega_{y} \downarrow \Omega_{x}}$ on the frame of discernment $\Omega_{x}$ is defined by:

$$
\begin{gathered}
m^{\Omega_{x} \Omega_{y} \downarrow \Omega_{x}}(A)=\sum_{B \subseteq \Omega_{x} \Omega_{y} / \operatorname{Proj}\left(B \downarrow \Omega_{x}\right)=A} m^{\Omega_{x} \Omega_{y}}(B) \\
\forall A \subseteq \Omega_{x}
\end{gathered}
$$

Where $\operatorname{Proj}\left(B \downarrow \Omega_{x}\right)=\left\{x \in \Omega_{x} / \exists y \in \Omega_{y},(x, y) \in\right.$ $B\}$. The inverse operation is a particular instance of vacuous extension. Consider a BPA $m^{\Omega_{x}}$ defined on $\Omega_{x}$. Its vacuous extension on $\Omega_{x} \Omega_{y}$ is defined by:

$$
m^{\Omega_{x} \uparrow \Omega_{x} \Omega_{y}}(B)= \begin{cases}m^{\Omega_{x}}(A) & \text { if } B=A \times \Omega_{y} \\ 0 & \text { otherwise. }\end{cases}
$$

$$
\forall A \subseteq \Omega_{x}
$$




\subsection{Belief and plausibility functions}

The belief $\mathrm{Bel}$ and plausibility $\mathrm{Pl}$ functions for a subset $A$ are defined as follows:

$$
\begin{aligned}
\operatorname{Bel}(A) & =\sum_{B \subseteq A} m^{\Omega}(B) \\
\operatorname{Pl}(A) & =\sum_{B \cap A \neq \emptyset} m^{\Omega}(B) \quad \forall A \subseteq \Omega, \forall B \subseteq \Omega
\end{aligned}
$$

$\operatorname{Bel}(A)$ is the degree to which the evidence supports $A$ and $P l(A)$ is the maximal degree of support that could be assigned to $A$ if there were more available evidence. $\mathrm{Pl}(A)$ may also be defined as the extent to which we fail to disbelieve the hypothesis of $A$. $[\operatorname{Bel}(A), P l(A)]$ can be viewed as the interval that describes the uncertainty of $A$. The functions $\mathrm{Bel}$ and $\mathrm{Pl}$, although being also function mapping events $\mathrm{A}$ into $[0,1]$ and mapping $\emptyset$ into 0 and $\Omega$ into 1 , do not fulfill in the general case the sub-additivity properties given for probability.

\subsection{Reliability assessment}

A proper reliability model using the TBM was introduced by Sallak et al. (2010) and further studied in (Aguirre et al. 2010, Aguirre et al. 2011). In these works, different points are treated concerning the elicitation of reliability masses, the representation of the system's configuration, the failure dependencies between components and a comparison with other methods is made. Expressed in words, the reliability analysis through the use of the TBM can be defined as follows:

1. A binary state is considered. Components' states and the system states are defined over a frame of discernment $\Omega=\left\{F_{i}, W_{i}\right\}$, with $i$ being a number that represents a component or $s$ for the system.

2. The reliability of each component is expressed in the form of a mass function $m^{\Omega_{i}}$ that maps the power set $2^{\Omega_{i}}=\left\{\left(F_{i}\right),\left(W_{i}\right),\left(F_{i}, W_{i}\right)\right\}$ to the interval $[0,1]$. The masses given to $F_{i}$ and $W_{i}$ represents, respectively, the belief on the Failure state and on the Working state. The mass given to $\left(F_{i}, W_{i}\right)$ represents the epistemic uncertainty, that is, a measure of the ignorance about a components state. The elicitation process of this masses is based on experts' opinions.

$$
\begin{aligned}
m^{\Omega_{i}}\left(\left\{F_{i}\right\}\right) & =f_{i} \\
m^{\Omega_{i}}\left(\left\{W_{i}\right\}\right) & =w_{i} \\
m^{\Omega_{i}}\left(\left\{W_{i}, F_{i}\right\}\right) & =1-w_{i}-f_{i} \\
i & =1,2 \ldots n
\end{aligned}
$$

Note that if $m^{\Omega_{i}}\left(\left\{W_{i}, F_{i}\right\}\right)=0$, the mass is called bayesian mass function and if this holds for every component $i$, then, the TBM model will yield the same results as the classical probability reliability analysis. Here there are two things to notice: first, this proves that probability theory is a particular case of the D-S theory (in our case we use the TBM interpretation). Second, the epistemic uncertainty is represented by the value assigned to $m^{\Omega_{i}}\left(\left\{W_{i}, F_{i}\right\}\right)$, therefore, this value being equal to zero means that only aleatory uncertainty is present for the given component.

3. The configuration of the system is then represented by a categorical mass function $m_{\text {Config }}^{\Omega}$. This type of mass is simply a mass containing only one focal element and represents the certitude that the truth can be found in this focal element. As it is consider that the system configuration is well known, this certitude is expressed by coding the truth table of the system configuration to a focal element and giving it a mass of 1. The focal element will contain every possible combined states and will act as a filter that keeps only the states representing the given system.

4. Once the reliability of components and the system's configuration are expressed by a mass function, all these masses are combined so as to obtain at the end the system's reliability. First, each reliability mass $m^{\Omega_{i}}$ must be extended to the product space $\Omega_{1} \Omega_{2} \cdots \Omega_{n} \Omega_{S}$ using Eq. (4). Then, all of the components' reliability masses and the system's configuration mass $m_{\text {Config }}^{\Omega}$ are combined using the conjunctive rule of combination (Eq. 2). Finally, the obtained mass is projected to the frame of discernment of the system's states $\Omega_{s}$ using Eq. (3). This process is represented by Eq. (8)

$$
m^{\Omega_{S}}=\left(\oplus_{i=1}^{n} m^{\Omega_{i} \uparrow \Omega_{1} \Omega_{2} \cdots \Omega_{n} \Omega_{S}} \oplus m_{C \text { onfig }}^{\Omega_{1} \Omega_{2} \cdots \Omega_{n} \Omega_{S}}\right)^{\downarrow \Omega_{S}}
$$

The system's reliability $R_{S}$ is expressed in terms of a belief and a plausibility measure obtained from the resulting mass $m^{\Omega_{s}}$ using Eqs. (5) and (6). It will be bounded by:

$$
R_{S} \in\left[\operatorname{Bel}\left(\left\{W_{S}\right\}\right), P l\left(\left\{W_{S}\right\}\right)\right]
$$

For further detail of the TBM reliability model, see Sallak et al. 2010.

\section{COMPUTATIONAL COST OF THE TBM MODEL}

The TBM reliability model and the classical probability reliability model have their differences. From one side, the TBM model is capable of treating epistemic 




Figure 1: Computational time as a function of the size of the system (launched in a server with $32 \mathrm{~Gb}$ of RAM and an octo dual core Opteron 8218)

uncertainty about the reliability of components, about the systems configuration and even about the dependencies between the failure of components. This capability is one of the most important things the TBM has to offer in comparison to the classical probability model, a proper treatment of epistemic uncertainty, not leaving a side off course the aleatory uncertainty. However, it is a capability that has a cost in terms of computational time. Indeed, the computational cost of the model grows exponentially with the size of the studied system.

From Eq. (7), it can be seen that the reliability mass $m^{\Omega_{i}}$ of each component is defined over 3 focal elements. As depicted in step 4 of section 2.5, each $m^{\Omega_{i}}$ is extended to the product space $\Omega_{2} \cdots \Omega_{n} \Omega_{S}$ and then, all the $m^{\Omega_{i}}$ are combined with each other $\left(\oplus_{i=1}^{n} m^{\Omega_{i} \uparrow \Omega_{1} \Omega_{2} \cdots \Omega_{n} \Omega_{S}}\right)$.

As each component is defined over three focal elements, and thanks to the nature of the conjunctive combination rule, the resulting mass after combination is defined over $3^{n}$ focal elements with each of the focal elements having from 2 to $2^{n+1}$ elementary events (defined over the product space $\Omega_{2} \cdots \Omega_{n} \Omega_{S}$ ).

To start with, doing the calculation by hand can be a pretty hard task for a system of as little as four components. The resulting mass would have 81 focal elements with up to 32 elementary events. As a response to this inconvenient, a Matlab TBM Toolbox has been created capable of doing a reliability analysis using the TBM model, hence, avoiding the tough task of doing this by hand. The toolbox makes a numerical and analytical analysis. However, as the computational cost grows exponentially (Figure 1) a clever way to combine the masses and treat the model has to be implemented.

In order to optimize the TBM operations and saving time and space, some computation algorithms were given in (Shafer et al. 1987, Almond 1995). The idea of these algorithms lies in doing local calculations in smaller product spaces so as to reduce the exponential growth of the TBM. For the case of reliability analysis, the idea of the algorithms is to divide the system in subsystems and to analyze the reliability of each subsystem to further calculate the reliability of the whole system. This approach can go as deep as needed and even divide the subsystems in more subsystems substantially extending the computational limit of the size of the system.

On the other hand, Smets (1993) introduced a different approach that propagates conditional belief functions over a directed acyclical network called belief network. The advantage is that the edges of the graphs are weighted by conditional belief functions and not by joint belief functions over a product space, thus, its a more optimal method in terms of computational cost. The approach is less general but, the loss of generality doesn't affect the reliability analysis, plus, its also more natural to asses conditional belief functions than joint probabilities over a product space. Using this method, fault trees analysis could be extended in a proper way to integrate belief functions to model the gates and the failure occurrences.

Smets (2002) introduced matrix calculus using the Möbius transformation to the TBM. This approach simplifies greatly the development of software and optimizes the computational time but, there is still the problem of the exponential growth.

To overcome the computational time limit, generalized expressions were defined so as to have a more efficient way to study the reliability of systems. These expressions can be used to study series-parallel, parallel-series.

\section{GENERALIZED EXPRESSIONS FOR SERIES-PARALLEL AND PARALLEL-SERIES SYSTEMS USING THE TBM}

The expressions were obtained using the method of minimal cuts and minimal paths. The advantage of this equations is that any system that can be represented as a parallel-series or series-parallel system can be studied easily and faster. This way, there is no need to go by the TBM operations each time you want to compute the reliability of a system, thus, the inherent exponential growth of the TBM is avoided.

The system success can be represented trough the use of minimal cuts $S_{\text {cut }}$ or minimal paths $S_{\text {path }}$ with the following equations:

$$
S_{\text {path }}=\bigcup_{i=1}^{N_{T}} \bigcap_{j=1}^{n\left(T_{i}\right)} W_{T_{i}(j)} \quad S_{\text {cut }}=\bigcap_{i=1}^{N_{C}} \bigcup_{j=1}^{n\left(C_{i}\right)} W_{C_{i}(j)}
$$

Starting from the generalized equations of the parallel and series systems (Table 1) and from the principle of minimal paths and minimal cuts (Eqs. 10), 
the generalized reliability expressions shown in table 2 are obtained. The system reliability will be bounded by $R_{s} \in\left[\operatorname{Bel}\left\{W_{S}\right\}, P l\left\{W_{S}\right\}\right]$. The following notation is used:

$N_{T} \quad \mathrm{Nb}$. of minimal paths in the system

$N_{C} \quad \mathrm{Nb}$. of minimal cuts in the system

$n\left(T_{i}\right) \quad \mathrm{Nb}$. of components in the $i_{t h}$ minimal path

$n\left(C_{i}\right) \quad \mathrm{Nb}$. of components in the $i_{t h}$ minimal cut

$T_{i} \quad$ Index set of the $i_{t h}$ minimal path

$C_{i} \quad$ Index set of the $i_{t h}$ minimal cut

Table 1: Reliability belief function of parallel and series systems with $n$ components

\begin{tabular}{|l|c|c|}
\hline & Parallel system & Series system \\
\hline $\operatorname{Bel}\left(W_{S}\right)$ & $1-\prod_{i=1}^{n}\left(1-m\left\{W_{i}\right\}\right)$ & $\prod_{i=1}^{n} m\left\{W_{i}\right\}$ \\
\hline $\operatorname{Pl}\left(W_{S}\right)$ & $1-\prod_{i=1}^{n} m\left\{F_{i}\right\}$ & $\prod_{i=1}^{n}\left(1-m\left\{F_{i}\right\}\right)$ \\
\hline
\end{tabular}

Table 2: Generalized reliability belief functions through the use of minimal cuts and minimal paths.

\begin{tabular}{|l|c|}
\hline \multicolumn{2}{|c|}{ Minimal cuts } \\
\hline $\operatorname{Bel}\left(W_{S}\right)$ & $\prod_{i=1}^{N_{C}}\left(1-\prod_{j=1}^{n\left(C_{i}\right)}\left(1-m\left\{W_{C_{i}(j)}\right\}\right)\right)$ \\
\hline $\operatorname{Pl}\left(W_{S}\right)$ & $\prod_{i=1}^{N_{C}}\left(1-\prod_{j=1}^{n\left(C_{i}\right)} m\left\{F_{C_{i}(j)}\right\}\right)$ \\
\hline $\operatorname{Bel}\left(W_{S}\right)$ & $1-\prod_{i=1}^{N_{T}}\left(1-\prod_{j=1}^{n\left(T_{i}\right)} m\left\{W_{T_{i}(j)}\right\}\right)$ \\
\hline $\operatorname{Pl}\left(W_{S}\right)$ & $1-\prod_{i=1}^{N_{T}}\left(1-\prod_{j=1}^{n\left(T_{i}\right)}\left(1-m\left\{F_{T_{i}(j)}\right\}\right)\right)$ \\
\hline
\end{tabular}

It has to be taken into account that the presented expressions are limited to series-parallel and parallelseries systems (Kuo 2001, pp. 13-16). In the case of repeated events, the correct way to proceed is to use disjoint cuts or paths. In this work, we use the minimal paths expressions for the series-parallel systems and the minimal cuts expressions for the parallel-series systems.

For the case were all of the components have the same reliability values $\left(m^{\Omega_{i}}\left(\left\{W_{i}\right\}\right)=w\right.$, $m^{\Omega_{i}}\left(\left\{F_{i}\right\}\right)=f$ ) and the system is symmetrical (e.g., fig. 2 , system b), the expressions are reduced to those shown in table 3.

Furthermore, if the reliability belief functions for the components are bayesian belief functions $\left(m^{\Omega_{i}}\left(\left\{W_{i}, F_{i}\right\}\right)=0, \quad \forall i=1,2 \ldots n\right)$, the system reliability will also be defined by a bayesian belief function and the following equality will hold: $\operatorname{Bel}\left(W_{S}\right)=$ $\mathrm{Pl}\left(W_{S}\right)$.
Table 3: Generalized reliability belief functions through the use of minimal cuts and minimal paths. Special case where the system is symmetric and its components have the same reliability

\begin{tabular}{|l|c|}
\hline \multicolumn{2}{|c|}{ Minimal cuts } \\
\hline $\operatorname{Bel}\left(W_{S}\right)$ & $\left(1-(1-w)^{n(C)}\right)^{N_{C}}$ \\
\hline $\operatorname{Pl}\left(W_{S}\right)$ & $\left(1-f^{n(C)}\right)^{N_{C}}$ \\
\hline \multicolumn{2}{|c|}{ Minimal paths } \\
\hline $\operatorname{Bel}\left(W_{S}\right)$ & $1-\left(1-w^{n(T)}\right)^{N_{T}}$ \\
\hline $\operatorname{Pl}\left(W_{S}\right)$ & $1-\left(1-(1-f)^{n(T)}\right)^{N_{T}}$ \\
\hline
\end{tabular}



(a)

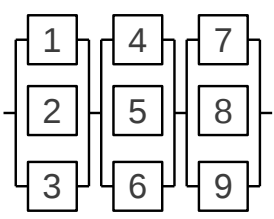

(b)
Figure 2: Reliability block diagram of the studied systems.

\section{APPLICATION}

In order to demonstrate the applicability of the expressions and the advances in computational time that can be achieved, a reliability analysis of the two different systems depicted in figure 2 is performed. For simplicity, all of the components have the same reliability, however the expressions can be used for the more general case where all of the components have different reliabilities.

System (a) is composed of three subsystems, two series subsystems and one series-parallel subsystem. Using the generalized expressions from table 2, the obtained belief and plausibility on the working state of the system is:

$$
\begin{aligned}
\operatorname{Bel}\left(W_{S}\right) & =\left(1-(1-w)^{2}\left(1-w^{2}\right)\right) w^{4} \\
& =w^{5}\left(w^{3}-2 w^{2}+2\right) \\
\operatorname{Pl}\left(W_{S}\right) & =\left(1-f^{2}\left(1-(1-f)^{2}\right)\right)(1-f)^{4} \\
& =(1-f)^{4}\left(f^{4}-2 f^{3}+1\right)
\end{aligned}
$$

System (b) is a parallel-series system, using the generalized expressions from table 3 , The belief and plausibility on the working state of the system is:

$$
\begin{aligned}
\operatorname{Bel}\left(W_{S}\right) & =\left(1-(1-w)^{3}\right)^{3} \\
\operatorname{Pl}\left(W_{S}\right) & =\left(1-f^{3}\right)^{3}
\end{aligned}
$$

\subsection{Comparison}

Using the Matlab TBM toolbox, the same results are obtained as in Eqs. (11) and (12) but, at a higher computational cost. For the system (a), the time of calculation is of approximately one hour and for system (b) it is of approximately ten minutes (launched in 
a server with $32 \mathrm{~Gb}$ of RAM and an octo dual core Opteron 8218). On the other hand, using the general expressions the computational cost is insignificant (a fraction of a second). The reliability of both of the proposed systems can be easily studied by hand using the proposed general expressions, whereas by the means of the TBM reliability model it would be an long task for systems of these sizes or bigger.

Note that thanks to the nature of the TBM reliability model, the computational time depends very little on the architecture of the system compared to the computational cost of the TBM. In fact, the computational time depends greatly on the size of the system, as shown in the application, a system of 8 components takes $\simeq 10 \mathrm{~min}$ and a system of 9 components takes $\simeq 1 h r$. Furthermore, a system of 10 components takes $\simeq 5 h r$. It can be noted that the computational time grows exponentially with the size of the system. In contrast, using a simple code that implements the presented generalized expressions, the computational limit is significantly higher. For example, a parallelseries system of 10 million components can still be studied in a fraction of a second.

\section{CONCLUSIONS}

Generalized reliability expressions for parallel-series and series-parallel systems using the TBM have been presented. The expressions represent a significant advance in terms of the computational limit of the theory. More precisely, they allow us to study systems with a considerably higher number of components. However, even if the TBM reliability model has a higher computational cost, it still represents a formal framework to continue the advances in the application of the TBM theory in the reliability analysis. Moreover, the model can be further optimized by using graphical methods based on local computations on reduced product spaces or by the implementation of conditional belief functions.

\section{REFERENCES}

Aguirre, F., M. Sallak, \& W. Schön (2010). Transferable belief model for incorporating failure dependencies in reliability analysis under data uncertainties. In Workshop on the Theory of Belief Functions. Brest, France.

Aguirre, F., M. Sallak, \& W. Schön (2011). Prise en compte des incertitudes dans les études de fiabilité : Comparaison entre le modèle de croyances transférables et les méthodes de simulations de Monté-Carlo. In Qualita 2011. Angers, France.

Almond, R. G. (1995). Graphical belief modeling. Chapmand and Hall.

Aven, T. (2010). Interpretations of alternative uncertainty representations in a reliability and risk analysis context. Reliability Engineering \& System Safety 96, 353-360.

Dempster, A. P. (1967). Upper and lower probabilities induced by multivalued mapping. Annals of Mathematical Statistics 38, 325-339.

Drouin, M., G. Parry, J. Lehner, G. Martinez-Guridi, J. LaChance, \& T. Wheeler (2009). Guidance on the
Treatment of Uncertainties Associated with PRAs in Risk-informed Decision making. Report, NUREG1855-V.1.

Kohlas, J. \& P. A. Monney (1995). A mathematical theory of hints: An approach to dempster-shafer theory of evidence. Lecture Notes in Economics and Mathematical Systems, Springer-Verlag. 425.

Kuo, W. (2001). Optimal reliability design: fundamentals and applications. Cambridge Univ Pr.

Moore, R. (1979). Methods and Applications of Interval Analysis. PA: SAIM Publ.

Oberkampf, W. L., J. C. Helton, C. A. Joslyn, S. F. Wojtkiewicz, $\&$ S. Ferson (2004). Challenge problems: uncertainty in system response given uncertain parameters, Volume 85.

Ruspini, E. H. (1986). The logical foundations of evidential reasoning. Technical note 408, SRI International, Menlo Park, Ca, US.

Sallak, M., W. Schön, \& F. Aguirre (2010). The Transferable Belief Model for reliability analysis of systems with data uncertainties and failure dependencies. Proceedings of the Institution of Mechanical Engineers, Part O: Journal of Risk and Reliability 4, 266-278.

Sentz, K. \& S. Ferson (2002). Combination of evidence in dempster-shafer theory. Technical report.

Shafer, G. (1976). A Mathematical Theory of Evidence. Princeton University Press.

Shafer, G., P. P. Shenoy, \& K. Mellouli (1987). Propagating belief functions in qualitative markov trees. International Journal of Approximate Reasoning 1, 349-400.

Smets, P. (1993). Belief functions: The disjunctive rule of combination and the generalized bayesian theorem. International Journal of Approximate Reasoning 9(1), 1-35.

Smets, P. (2002). The application of the matrix calculus to belief functions. International Journal of Approximate Reasoning 31(1-2), 130.

Smets, P. \& R. Kennes (1994). The transferable belief model. Artificial Intelligence 66, 191-243.

Walley, P. (1991). Statistical reasoning with imprecise probabilities. New York : Chapman and Hall.

Zadeh, L. (1965). Fuzzy sets. Inform Control 8, 338-353. 